\title{
The Cardiorenal Syndrome
}

\author{
Mitchell H. Rosner, ${ }^{1}$ Anjay Rastogi, ${ }^{2}$ and Claudio Ronco ${ }^{3}$ \\ ${ }^{1}$ Division of Nephrology, University of Virginia Health System, P.O. Box 800133, Charlottesville, VA 22908, USA \\ ${ }^{2}$ Division of Nephrology, University of California Los Angeles, 200 UCLA Medical Plaza, Suite 365B, Los Angeles, CA 90095, USA \\ ${ }^{3}$ Department of Nephrology Dialysis \& Transplantation and International Renal Research Institute (IRRIV), San Bortolo Hospital, \\ Viale Rodolfi 37, 36100 Vicenza, Italy
}

Correspondence should be addressed to Mitchell H. Rosner, mhr9r@virginia.edu

Received 12 May 2011; Accepted 12 May 2011

Copyright ( 2011 Mitchell H. Rosner et al. This is an open access article distributed under the Creative Commons Attribution License, which permits unrestricted use, distribution, and reproduction in any medium, provided the original work is properly cited.

This special issue of the International Journal of Nephrology is the first nephrology journal edition devoted solely to the discussion of the cardiorenal syndrome and represents a landmark state-of-the-art discussion on such pathological entity. The cardiorenal syndrome (CRS) was first officially defined at a consensus conference of the Acute Dialysis Quality Initiative in 2009 [1]. This definition was made in the attempt to characterize and classify the various connections between acute and chronic heart and kidney disease. Although well known, heart and kidney interactions had not been clearly defined in the past nor were they completely elucidated and classified. The consensus conference defined 5 forms of heart-kidney interaction that would lay the foundation for both a common language in describing patients suffering from these syndromes as well as forming a schema for further research. The consensus conference recognized that communication between the heart and kidneys occurs through a variety of pathways that in the healthy state modulate cardiac output, vascular tone, maintenance of volume state, and excretion of waste products. However, a change in the performance of one of these organs elicits a cascade of mediators that affects the other and leads to a spiral of mutual organ dysfunction.

The five forms of the CRS include (1) type I CRS where acute heart failure is directly associated with acute kidney injury (AKI); (2) type II CRS in which chronic heart failure is associated with chronic kidney disease (CKD); (3) type III CRS where AKI is associated with acute heart failure; (4) type 4 CRS in which the driving factor of CKD is associated with chronic heart failure; (5) type 5 CRS where there is concomitant development of both kidney and heart failure.
The importance of having such a classification scheme is due to the fact that both cardiovascular disease (CVD) and CKD are highly prevalent and overlapping conditions. For instance, it is estimated that 1 in 3 adults in the United States (US) (>100 million persons) has a diagnosis of some form of CVD (hypertension, coronary heart disease, heart failure (HF), stroke, or congenital heart disease), and nearly $13 \%$ of the US population has been estimated to have some form of CKD $[2,3]$. Much of this disease burden occurs in the same patient as acute, and chronic abnormalities in cardiac function are associated with an increased risk for kidney injury, and patients with both AKI and CKD have a large burden of CVD [2-6]. For example, in the ADHERE study database of hospital admission for acute decompensated heart failure (ADHF), $27.4 \%, 43.5 \%$, and $13.1 \%$ of all patient admissions were found to have mild, moderate, or severe kidney dysfunction, respectively [6]. It is thought that the overlap between CVD and kidney dysfunction represents common pathophysiological processes that interact in deleterious ways to promote a cycle of organ dysfunction. These critical, dynamic, and bidirectional interactions include varied pathophysiological pathways that are discussed in this special edition. These pathways range from hemodynamic interactions to widespread inflammation that affects multiple organ systems. As with any newly described syndrome, the questions outnumber the answers and papers in this edition attempt to offer both hypothesis for how organ crosstalk may occur between the heart and kidney, as well as describe important diagnostic aids for CRS, and potential therapies that may improve cardiac and kidney function and define questions that will require further elucidation. 
Given both the prevalence and the impact of the CRS, it is clear that there is a mandate for more research in this evolving area. This journal edition represents a beginning on the road to answers.

\section{Mitchell H. Rosner \\ Anjay Rastogi \\ Claudio Ronco}

\section{References}

[1] C. Ronco, P. McCullough, S. D. Anker et al., "Cardio-renal syndromes: an executive summary from the consensus conference of the Acute Dialysis Quality Initiative," Contributions to Nephrology, vol. 165, pp. 54-67, 2010.

[2] “Cardiovascular disease 2008," http://www.americanheart.org.

[3] J. Coresh, E. Selvin, L. A. Stevens et al., "Prevalence of chronic kidney disease in the United States," Journal of the American Medical Association, vol. 298, no. 17, pp. 2038-2047, 2007.

[4] R. N. Foley, A. M. Murray, S. Li et al., "Chronic kidney disease and the risk for cardiovascular disease, renal replacement, and death in the United States medicare population, 1998 to 1999," Journal of the American Society of Nephrology, vol. 16, no. 2, pp. 489-495, 2005.

[5] C. M. Logar, C. A. Herzog, and S. Beddhu, "Diagnosis and therapy of coronary artery disease in renal failure, end-stage renal disease, and renal transplant populations," American Journal of the Medical Sciences, vol. 325, no. 4, pp. 214-227, 2003.

[6] J. T. Heywood, G. C. Fonarow, M. R. Costanzo, V. S. Mathur, J. R. Wigneswaran, and J. Wynne, "High prevalence of renal dysfunction and its impact on outcome in 118,465 patients hospitalized with acute decompensated heart failure: a report from the ADHERE database," Journal of Cardiac Failure, vol. 13, no. 6, pp. 422-430, 2007. 


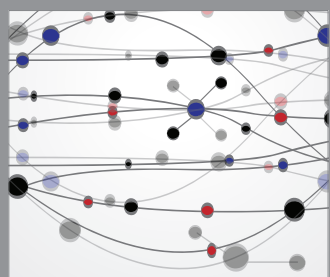

The Scientific World Journal
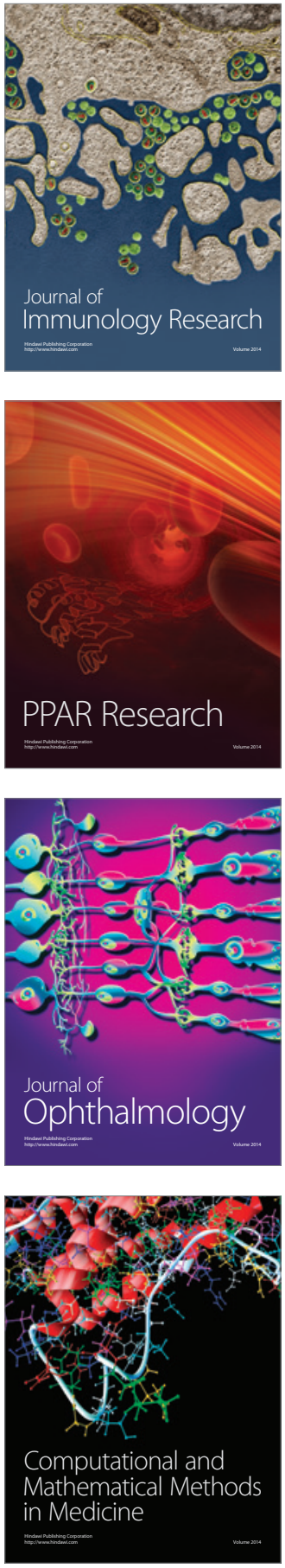

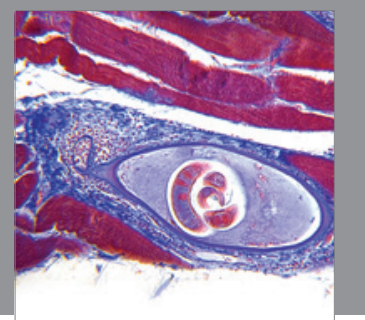

Gastroenterology

Research and Practice
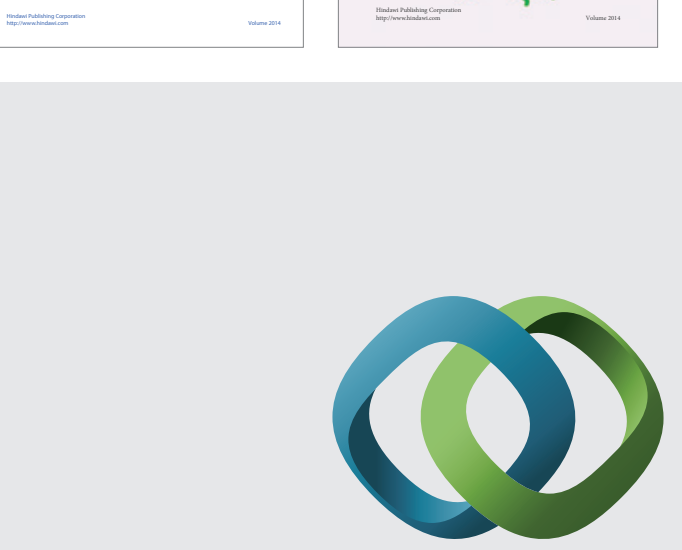

\section{Hindawi}

Submit your manuscripts at

http://www.hindawi.com
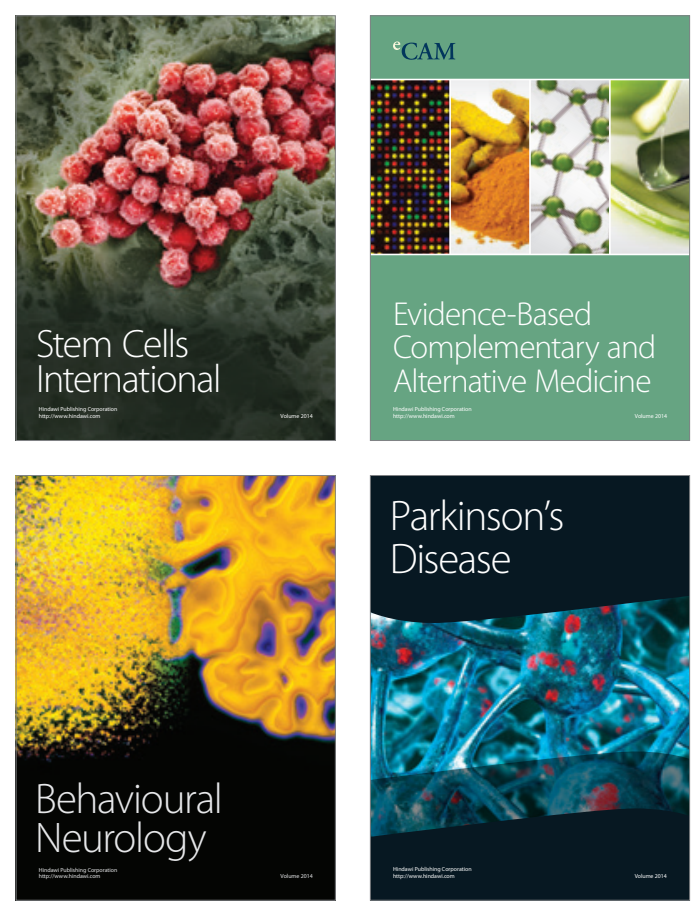

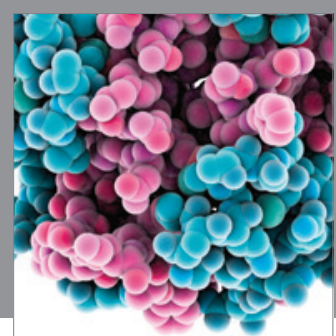

Journal of
Diabetes Research

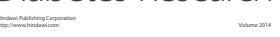

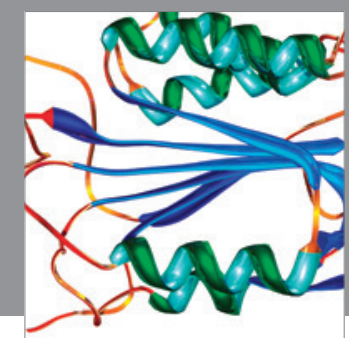

Disease Markers
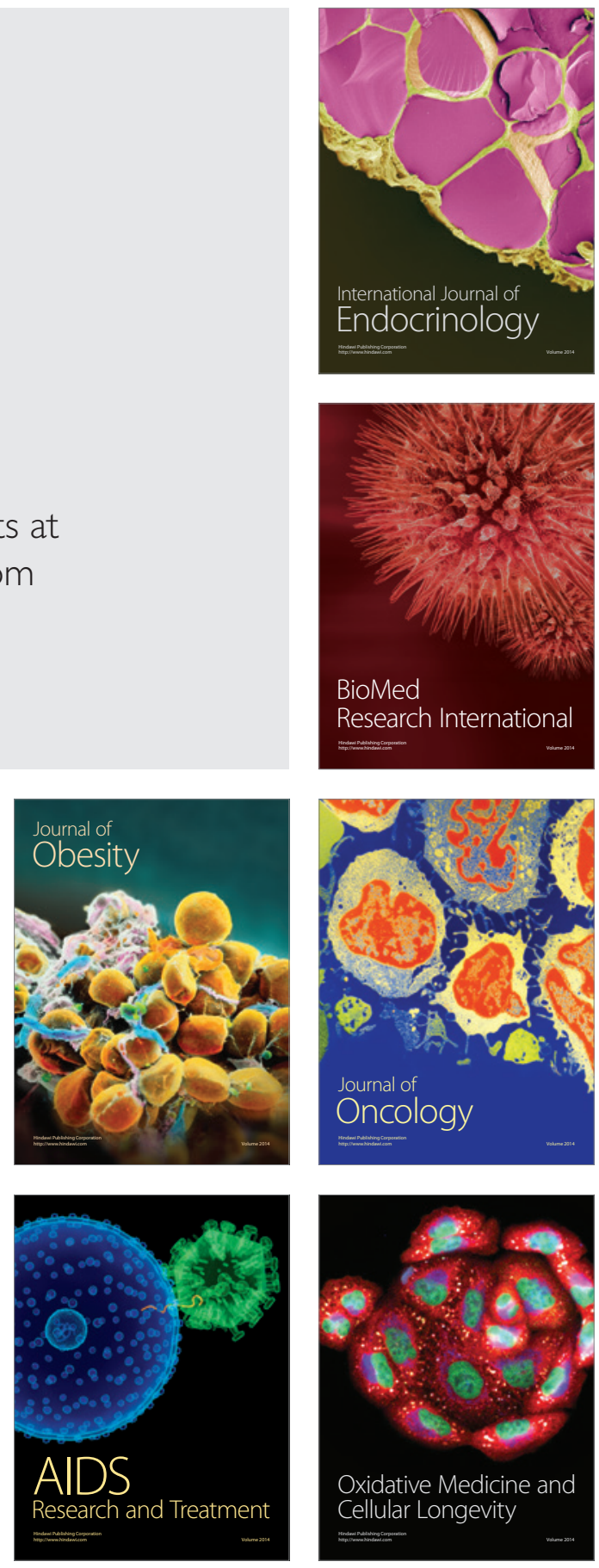